\title{
Decision Making Process for Prioritizing Replacement of Medical Equipment Considering Non-technical Factors
}

\author{
Tariq Alqahtani \\ Department of Medical Equipments Technology, College of Applied medical Science, Majmaah \\ University, 11952, Majmaah, Saudi Arabia \\ t.alqahtani@mu.edu.sa
}

\begin{abstract}
The decision to replace a large number of medical equipments is, necessarily, a multicriteria one. The author work set out to look into the current system being used at the level of the Ministry of Health in the Kingdom of Saudi Arabia to prioritize the replacement of medical equipment. That system, as it operates, is inefficient and does not utilize available resources competently. It relied mostly on technical data and specifications of medical equipment on the replacement list. The author believes that, relevant nontechnical data should be used to enhance the quality of replacement prioritization decision. Current decision support systems do not take this into account, this represent a shortfall in existing knowledge. A review of relevant literature of existing Multi-Criteria Decision Making (MCDM) systems, both in the healthcare and other industries, was undertaken. This included AHP, PROMETHEE, VIKOR and Factorial Surveys (vignette). The author chose a new hybrid approach; it included the combination of AHP and vignette. This allowed weighting of non-technical data (identified as attributes throughout the thesis work) and incorporation of responses as obtained from respondents to the vignettes. The hybrid system was tested on the ground by applying it to theoretical medical equipment tender. The author found that, the use non-technical factors have merits in deciding on medical equipment priority replacement list. It is not certain if the newly developed hybrid system would be robust enough to solve the shortcomings of the existing system.
\end{abstract}

Keywords: Decision-making, Replacement, Medical Equipment, Non-technical, Biomedical

\section{Introduction}

According to Abbas (1993) decision-making is probably the most important function of any healthcare manager, as it is the variable upon which the success or failure of any organization depends. Hipkin and Lockett (1995) emphasized this view when they concluded that, replacement decisions are no longer a tactical exercise, but rather a strategic function. The mechanism of decision making to prioritize replacing medical equipments at present is inadequate. It fails to take into account factors such as: fast-paced advances in development of medical technology, geographic aspects, and environmental issues, exact need of technology services, economic aspect, and end users or technician assessment. At times, it was noted that there is no consistency between the clinical need for the given equipment and deciding on the priority to replace it. Furthermore, political issues and points of views can influence the members of the 
committee, which obviously is not conducive to the work and the decision making of those members. The author, firmly, believes that, non-technical factors, as stated above, are very important to give the optimal replacement priority decision. Unfortunately, based on the personal experience, there is chronic insufficiency in using data generated from such factors when prioritizing medical equipment replacement at local and national levels. In the context of priority, there are no clear rules or guidelines laid out by the ministry that can be followed. This reflects on the accuracy, quality of decision, healthcare services, and optimal utilization of the replacement budget. The majority of research carried out in the area of priority policies for equipment replacement or equipment replacement in general, has been in the non-medical sector; that is, in the industrial sector. It would be difficult to implement the current applications found in the literature without some changes and modifications to the methods used. Such modifications would need to take into account the added needs for safety of patients and that of healthcare users. The Ministry of Health oversees different hospitals and medical centers. They are located in variable geographic areas, serve different demographics and provide various services at different levels of specialism. The end users in these institutes have users of different levels of expertise and needs for medical equipments. These variable factors necessitate the need to expand the spectrum of factors that can affect the replacement priority decision so that, it is more generalized and covers all aspects of the replacement. Added to all this is the variety of judgments and views expressed by healthcare managers, replacement committee members and others at the executive level. Those stakeholders have a collective sum of wealth of knowledge and experience which, need to be taken into account when prioritizing and deciding on replacement lists. The author argues that, these two aspects of non-technical data need to be integrated in the decision making process. To accomplish this, a new assessment method would be required. This would, likely, rely on a scoring system and a method of capturing qualitative and non-technical data. The current methods reviewed by the author do not accommodate the requirements as stated above. This creates the need to bring about a tool to bridge the existing gap. Such tool should be flexible and adaptable to the needs of system for which it was created. Furthermore, the outcomes or results should be accurate, reliable and measurable. The author decided to use a combined approach to fill in the gap. Such approach, in brief, integrates a scoring system such as AHP and factorial surveys, further details below. The author believes that using methods that incorporate factorial surveys (vignettes) in engineering management, especially in the field of medical equipment management, may be an interesting and useful approach. This is because they enable incorporation of experiences of sociological and engineering perspectives. Furthermore, intersection and conflicts between decisions can be avoided, as the final decision is likely to represent and satisfy the views of most participant decision-makers. Finally, decision based on inadequate information often lead to budget mismanagement and allocation losses and this, ultimately, affects the quality of health services offered. Makis and Jardine (1992), Desa and Chirster (2001), Tanchoco and Leung (1987), and Hartman (2001) agreed that; traditional equipment replacement and traditional economic replacement analysis was, generally, based on models, which focused on maintenance information, and parameters of length of use and the number of repairs or failures. The optimal replacement policy was to maximize the terminal wealth of equipment utility. Moreover, Suzuki and Pautsch (2005) identified age of industrial assets as one of the factors onto which replacement policy can be based. Tomsovic and Baer (1996) added that the traditional approach for industrial device replacement is based on a vendor's recommendations, and is commonly for fixed time intervals only. Their research focused on the theoretical framework for systematically evaluating 
Tariq Alqahtani; Decision Making Process for Prioritizing Replacement of Medical Equipment Considering Non-technical Factors. Journal of Biomedical Engineering and Medical Imaging, Volume 6, No 3, June (2019), pp 1-5

equipment condition. The process of replacement of medical equipment is a complex one. Campbell (1994) found in his study that, replacements particularly of equipment are mostly dictated by physicians or regulators. In addition, the decision to replace any medical equipment depends largely on the number of many different factors. Rising operating costs and utilization were ranked as less important in replacement decision. In addition the author shows in the result that the technological obsolescence was the most important factor for equipment replacement, when some equipment were replaced before becoming obsolete during the demand of new technology and improve the quality of the diagnoses and treatment. This paper has been used the technical factors as a base of replacement priority while the author's research makes the non-technical factors as a base of replacement priority decision parallel with the analysis of decision maker opinion. Fennigkoh (1992) proposed the use of a scoring system to recommend and prioritize equipment replacement based on a numerical output. This study has a good argument for determining the priority list, but adding more attributes. This may be useful and the number of the factors should be considered in the issue of more decision accuracy.

\section{Materials and Methods}

The author decided to focus on studying a limited number of approaches. Through these reviews and studies, the author decided to utilize two particular methodologies. These are Analytical Hierarchy Process (AHP) and Factorial Surveys (FS). The author found that these two applications were among the easiest and most appropriate to use given the core question of his work i.e. measurement and analysis of the impact of non-technical factors.

Furthermore, AHP and FS were the two closes applications that conveyed what this work set out to achieve. Additionally, the proposed combination of AHP and FS is very likely to be useful no only in the medical equipment field but in other fields as well.

\section{Results}

The design of the new approach was achieved through the integration of AHP and FS. This was done in order to analyze and evaluate two major points in this research. These are as follows: The first point is the assessment of the impact of non-technical information on decision making. Also, understanding the sub factors that make up these non-technical data. This would allow the evaluation of which of these sub factors has more impact or weight that affects the outcome of the decision-making process. AHP was found to be the most appropriate method to achieve this goal, hence it was chosen. The second point is the understanding and utilization of the experience of engineers, medical personnel and top executive decision-makers in the purchase and replacement of ME. Such experience with an independent view and a totally different perspective on how to weigh the impact of non-technical factors must be evaluated. Understandably, to do so there must be a different and non-numerical way of evaluation as a human agent involved here is difficult to control. For this reason, factorial surveys or vignettes were chosen. The author used the highest weight score factors in the same level of hierarchy as the vignette items. This gave the decision makers a way to identify which factors have more emphasis and thus more effect. This then would lead to the appropriate decision making.

\subsection{Solution Development}

This research introduced a decision support system, which will first aid the end user in narrowing the choice set, and then assist in making the final choice. The foundation of this DSS includes a synergistic 
combination of two powerful analytic tools, AHP and vignettes. The resulting benefits from using this DSS include both a better understanding of the user's decision-making process and valuable assistance in making a priority based on the inclusion of nontechnical factors.

\subsection{Combine AHP and Vignettes}

As stated earlier, prioritizing replacement of medical equipment on the final replacement list is an important decision to make. This is due to the direct impact on the quality of healthcare services, the longterm investment in healthcare infrastructure and the overall success of healthcare services. Furthermore, as demonstrated, the process of prioritization is complex, multi-factorial and multi-person problem. It can be approached and solved by a systematic and logical approach to assess priorities based on the inputs of several tasks and information from different functional issues within the health services.

\section{Conclusion}

The hypothesized strategy is to use both AHP and vignette to support the prioritized choice replacement. This approach has some features of a skilled system in that; it relies on experts to specify the essential attributes as well as their hierarchy. In addition, all necessary information can be collected for all possible substitutions and branded in sets according to the definite traits.

\section{REFERENCES}

[1] Abbas J. Ali. "Decision-Making Style, Individualism and Attitudes toward Risk of Arab Executives". International Studies of Management \& Organization, Vol. 23, 1993

[2] Hipkin, I. B. and Lockett, A. G.(1995), “Study of Maintenance Technology Implementation”, Omega, International Journal of Management Science, Vol.23, Issue 1, pp: 79-88

[3] Makis V., \& Jardine, “Optimal Replacements policy for a general model with imperfect Repair" Journal of Operational Research Society. Vol. 43, no. 2, pp. 111-120

[4] Desa MI., \& Christer AH., "Modelling of the absence of the data: a case study of fleet maintenance in developed country", Journal of the Operational Research Society (2001) 52,247-260

[5] Tanchoco J.M.A., \&. Leung L.C., “An Input- Output Model for Equipment Replacement Decisions”, Engineering Costs and Production Economics, Volume 11, Issue 1, April 1987, pp: 69-78

[6] Hartman J.C.,"An economic replacement model with probabilistic asset utilization", 2001, IIE transactions (2001) $33,717-727$

[7] Suzuki Y., Pautsch G.R., "A vehicle replacement policy for motor carriers in an unsteady economy", Transportation Research part a 39(2005) 463-480

[8] Tomsovic, K. and Baer, B. (1996), "Methods of approximate reasoning for power system equipment condition and reliability analysis", Proceedings of the 1996 IEEE International Conference on Intelligent Systems Applications to Power Systems, pp: 310-315 
Tariq Alqahtani; Decision Making Process for Prioritizing Replacement of Medical Equipment Considering Non-technical Factors. Journal of Biomedical Engineering and Medical Imaging, Volume 6, No 3, June (2019), pp 1-5

[9] Campbell, C., "Hospital Plant and Equipment Decisions: A Survey of Hospital Financial Managers", Hospital \& Health Services Administration. Winter; 39(4); 538-58

[10] Fennigkoh, L. 1992 Jan, “Medical Equipment Replacement Model” Journal of Clinical Engineering. 17(1):43-7

[11] Saaty T., "The Analytic Hierarchy Process", 1980 NY, McGraw Hill Taylor, K. "A medical Equipment Replacement Score System", Journal of Clinical Engineering. January/March 2005 - Volume 30 - Issue 1 - pp: 37-41

[12] Taylor, K. “A medical Equipment Replacement Score System”, Journal of Clinical Engineering. January/March 2005 - Volume 30 - Issue 1 - pp: 37-41 